\title{
Socio-economic inequalities in testicular cancer survival within two clinical studies
}

Ula Nur ${ }^{1}$, Bernard Rachet ${ }^{1}$, Mahesh KB Parmar ${ }^{2}$, Mathew R Sydes ${ }^{2}$, Nicola Cooper ${ }^{3}$ Sally Stenning ${ }^{2}$, Graham Read ${ }^{4}$, Tim Oliver ${ }^{5}$, Malcolm Mason ${ }^{6}$, Michel P Coleman ${ }^{1}$

1 Cancer Research UK Cancer Survival Group, Non-Communicable Disease Epidemiology Unit, London School of Hygiene and Tropical Medicine, Keppel St, London WC1E 7HT, UK

2 Cancer Group, MRC Clinical Trials Unit, Aviation House, 125 Kingsway, London WC2B $6 \mathrm{NH}$

3 National Cancer Intelligence Centre, Social and Health Analysis and Reporting Division, Office for National Statistics, (Room FG/114) 1 Myddelton Street, London EC1R 1UW, UK

4 Royal Preston Hospital, Preston, PR2 9HT

5 Barts cancer institute, Barts and the London School of Medicine, Queen Mary University London, EC1M 6BQ

6 Cardiff University School of Medicine, Cardiff CF14 4XN

Funding: Cancer Research UK, UK Medical Research Council funds (MP, MS, SS).

Length: 2,348 words, 2 Tables, 2 Figure, 19 references.

\section{Correspondence}

Dr Ula Nur PhD, Lecturer in Cancer Survival, Cancer Research UK Cancer Survival Group, Department of Non-Communicable Disease Epidemiology, London School of Hygiene and Tropical Medicine, Keppel St, London WC1E 7HT, UK, +44 207927 2091, F + 44207436 4230, ula.nur@,1shtm.ac.uk 


\section{ABSTRACT}

Background: Testicular cancer is the most common cancer in men under 35 years of age, and has the highest survival for adult male malignancies. Despite the fact that survival is very high, there is evidence that survival differs between socio-economic groups.

Methods: We analyzed survival patterns for 1606 testicular cancer patients diagnosed during 1984-2001 and recruited to one of two clinical studies. The first was a surveillance study to determine relapse-free survival after orchidectomy in 865 patients with stage I nonseminomatous germ-cell testicular cancer diagnosed during 1984-1991 (TE04). The second study was a trial in which 1174 men with stage I seminomatous germ-cell tumours were randomised to receive radiotherapy or one injection of carboplatin between 1996 and 2001, (TE19). The number of men available for analysis from these two studies was 578 and 1028 respectively. We followed these patients up for their vital status, and assigned them an ecological measure of deprivation. Crude and relative survival were estimated at 5 and 10 years by socio-economic deprivation.

Results: No significant socio-economic gradient was seen: $1.3 \%$ (95\% CI $-0.3 \%$ to $3.1 \%)$ at 5 years and $2.1 \%(95 \% \mathrm{CI}-0.5 \%$ to $4.7 \%)$ at 10 years.

CONCLUSION: We conclude that, given equal treatment at a given stage of disease, survival from testicular cancer does not depend on socio-economic status. This suggests that the socio-economic gradient in testicular cancer survival in the general population is more likely to be attributable to health care system factors than to personal or socioeconomic factors in the men themselves.

Keywords: cancer survival, deprivation, cancer registries, testicular cancer, relapse, carboplatin. 


\section{INTRODUCTION}

Cancer survival in England and Wales differs between socio-economic groups for most adult cancers [1], and in many populations [2]. 5-year survival for adults from deprived areas was significantly lower than that of patients from affluent areas for most of the major 47 cancers [3]. Despite the fact that survival from testicular cancer is high, socioeconomic differences in survival were demonstrated for men diagnosed in England and Wales during the early 1970s [3], after adjustment for background mortality with life tables specific for each socio-economic group. Further evidence of socio-economic inequalities was confirmed in men with testicular cancer diagnosed during the late 1990s [4]. Differences in stage at diagnosis and access to treatment partly explain the socio-economic inequalities in cancer survival, but it is less clear why these differences arise [5].

Patients who take part in cohort studies or clinical trials fit strictly defined eligibility criteria and receive the same mandated treatment and follow-up, with close adherence to the study protocol. One would not expect the treatment received within each trial arm to vary between socio-economic groups, because the socio-economic status of the men was unknown at recruitment to the cohort study or randomisation in the trial.

The aim of this study was to measure any socio-economic differences in survival among men with testicular cancer recruited to two clinical studies. A socio-economic survival gradient within these study populations would imply that biological factors explain the survival gradient in the general population, while the absence of such a gradient would imply that access to treatment or other healthcare system factors are more likely to explain the inequalities in survival in the general population.

\section{MATERIALS AND METHODS}

The Medical Research Council (MRC) Clinical Trials Unit (CTU, formerly the MRC Cancer Trials Office) conducted the original testicular cancer studies, TE04 and TE19 (ISRCTN27163214).

The TE04 was a prospective single-arm cohort study which aimed to determine the rate of relapse and its predictive histological criteria among patients treated by orchidectomy alone for stage I nonseminomatous germ-cell testicular tumour (NSGCT). The patients were recruited from 16 United Kingdom centres and one Norwegian centre between January 1984 and October 1991, and attended follow-up assessment at monthly intervals for the first year, every 2 months for the second year and every 3 months for the third year, and regularly thereafter. The MRC Clinical Trials Unit constructed a dataset on a total of 865 men, of which 768 were registered in England or Wales between January 1984 and October 1991. Overseas patients and patients resident in Scotland were excluded because no information on their postcode of residence was available. Results for the first 396 men recruited between January 1984 and October 1987 confirmed the effectiveness of surveillance for the management of stage 1 NSGCT and identified a group of patients with high risk of relapse on histological criteria [6].

In the TE19 trial, 1477 patients from 70 hospitals in 14 countries with stage 1 seminomatous germ-cell tumours were randomly assigned by the MRC Clinical Trials Unit or the EORTC (European Organisation for Research and Treatment of Cancer) to receive either radiotherapy or one injection of carboplatin following orchidectomy. Relapse-free survival rates were compared between the trial arms. Carboplatin proved to 
be an effective adjuvant treatment and similar in outcome to radiotherapy [7] with respect to relapse rates. All non-UK patients were excluded, and a final dataset on 1174 men of whom 1112 were resident in England and Wales was prepared for analysis.

The Office for National Statistics (ONS) flagged patients on the National Health Service Central Register and provided information on their vital status (alive, dead, emigrated or lost to follow-up) up to 31 December 2008. The ONS also provided the postcode of the patient's residence at diagnosis, from which they were assigned to one of five deprivation categories (from 1 'most affluent' to 5 'most deprived'). Individual information was not available on the socio-economic status of these cancer patients; instead the Carstairs index [8], an ecological measure of deprivation based on four census-derived variables at the level of the census enumeration district (ED), was used to assign a deprivation category to patients diagnosed 1984-1995. The deprivation category was based on the 1981 census for men diagnosed 1984-1985 and 1991 census for men diagnosed 1986-1995. One of the four Carstairs components was changed in the 2001 census, and therefore was not comparable to that used in 1981 and 1991. The ONS introduced the Indices of Multiple Deprivation (IMD) in 2000. This new index is mostly based on routine administrative data and is regularly updated. It has already been shown that the choice of the deprivation index has little impact on the deprivation gap [9]. The ONS also changed the geographic level enumeration district (ED-mean population 450) to the larger but more socially homogenous level of the Lower Super-Output Areas (LSOA) (mean population 1 500) in 2001. For those diagnosed during 1996-2001, deprivation categories were defined from the income domain score of the (IMD2004) [10] using administrative data of the 34378 LSOAs in England. For patients in Wales, we used the equivalent Welsh index [11].

Of the 1880 patients resident in England or Wales initially considered for analysis, a further 274 patients (190 from TE04 and 84 from TE19) were excluded, either because

their postcode was missing, which meant that their socio-economic status could not be defined, or because their vital status was unknown i.e. patients who were not known to be dead, but whose records could not be traced to enable 'flagging' by the end of follow-up time, or because the record failed ONS validity checks i.e. one of the mandatory fields required by ONS was not correct (Figure 1).

Patients consented to join both studies. Multi-Centre Research Ethics Committee approval for this additional use of the data was obtained from West Midlands MREC for TE19 in 2006 and the MREC for Wales for TE04 in 2005. Approval was obtained for this study from the Ethics Committee of the London School of Hygiene and Tropical Medicine.

\section{Statistical analyses}

The men were followed up for at least five years to the end of 2008, the last year for which vital status was available through ONS. Survival time was calculated as the time between the date of diagnosis and the earliest of the date of death or 31 December 2008. Relative survival was estimated using the maximum-likelihood approach for individual data [12]. Relative survival is the ratio of the observed probability of survival in a cancer population to the expected probability of survival (background) in the general population with the same age, sex, geographic area, calendar period, and deprivation. This approach does not rely on the accurate reporting of cause of death, and is widely used in estimating population based survival. We report relative survival at 5 and 10 
years by deprivation category. The background mortality varies widely by age, sex, socioeconomic group and Government Office Region (GOR). For 1981-2005, background mortality was represented by complete life tables defined by sex, single year of age, calendar year, deprivation category and GOR [13]. Because of the unavailability of life tables by socioeconomic group and GOR for the years after 2005, the 2005 life tables were used for deaths that occurred during 2006-2008.

Socio-economic inequality in survival (the 'deprivation gap') was quantified as the fitted difference $(\%)$ between relative survival in the most deprived and most affluent groups in the study population, estimated with variance-weighted linear regression [14] applied to all the deprivation-specific survival estimates. We describe the deprivation gap as negative if relative survival is lower in the most deprived group. We then compared this gap with that in the general population of England for men diagnosed with testicular cancer during 1984-2001.

\section{RESULTS}

The distribution of socio-economic status among the 1606 men included in the analysis from the two studies was strikingly similar to that of men with testicular cancer in the general population of England and Wales: $16 \%$ in the most deprived group and $21-22 \%$ in each of the other four groups (Table 1). The distribution by deprivation was also similar in both studies. Around $70 \%$ of men were less than 40 years of age at diagnosis, almost $20 \%$ aged $40-49$, and only around $10 \%$ were aged 50 or over. The distribution by deprivation and age was very similar to that of all men diagnosed with testicular cancer in England and Wales during 1984-2001.

Relative survival in the study population was high and similar across the five deprivation groups. This ranged from $100 \%$ to $97 \%$ at 5 years, and from $100 \%$ to $96 \%$ at 10 years (Table 2). The fitted difference in survival between the most deprived and the most affluent groups in the combined study population was $1.4 \%$ five years after diagnosis and $2.2 \%$ ten years after diagnosis, meaning that the more deprived groups tended to have a higher survival than the 'most affluent' (Table 2, Figure 2). Neither result was statistically significant (Table 2). By contrast, survival was lower among the more deprived groups in the general population of England and Wales for men diagnosed during 1984-2001. The gap remained unchanged at $-3.1 \%(95 \% \mathrm{CI}-4.0 \%$ to $2.1 \%)$ at 5 years and $-3.1 \%(95 \% \mathrm{CI}-4.2 \%$ to $-2.1 \%)$ at 10 years (Table 2$)$.

\section{CONCLUSION}

Survival from testicular cancer was the highest among all malignancies in adult males in England and Wales throughout the period 1986-1999, exceeding 90\% ten years after diagnosis [4]. A significant deprivation gap in survival has been observed in the general population since the 1970 s, although the gap decreased substantially from $13 \%$ in the 1970 s to around 3\% during 1984-2001. This decrease in the deprivation gap appears to reflect a 'ceiling' effect, following the substantial improvements in survival associated with the introduction of platinum-based chemotherapy in the $1970 \mathrm{~s}$, in that there is little further room for increase in survival among men in the most affluent group. By contrast, in these two studies, affluent patients did not do better than the most deprived, and survival (as expected for patients diagnosed with stage I disease) exceeded $95 \%$ even ten years after diagnosis. A recent review of patients whose management was complex (Oliver RTD, Queen Mary University London) suggests that much of the remaining risk of death relates to the wide variety of clinicians to whom atypical metastatic 
manifestations of this disease can present, ranging from orthopaedic surgeons, gastroenterologists, thoracic surgeons, endocrinologists, neurologists and neurosurgeons, none of whom is routinely involved in the Multidisciplinary Team management of testicular cancer [15]. If improvement is to be made in their management, it may be necessary to develop some form of annual confidential review of deaths, as occurs with Maternal Mortality to give a global feedback where failure is occurring, as they often reveal problems involving four or more different clinicians.

Because of the excellent prognosis of patients with testicular cancer and the relative youth of the testicular cancer patients, only 72 deaths occurred among the 1606 patients included for analysis, all followed up for at least 5 years. The small number of deaths within each deprivation group was the major limitation of this study. This prohibited the estimation of relative survival and its confidence intervals with the available statistical methods for some of the deprivation categories with either none or very few deaths. All the men in the two studies had stage 1 testicular cancer.

Detailed information was available on treatment, socio-economic deprivation and vital status on 1606 patients in one cohort study and one randomised controlled trial. Allocation of patients to treatment in clinical studies and randomisation of patients in randomised trials ensures that patients follow the treatment policy stated in the study protocol, regardless of their socio-economic status. There was also no difference between the two trials in prognosis (all patients included with stage 1 testicular cancer), or by treatment within each of the analysed studies. In the TE04 study, all patients were treated by orchidectomy only prior to relapse, whereas in the trial (TE19), no evidence was found in favour of a difference in outcome between carboplatin and radiotherapy [7].

Both five-year and ten-year survival were higher among patients recruited to one of the two studies than among testicular cancer patients in England and Wales in the same period. This is an expected finding, in part because cancer patients taking part in clinical trials generally have higher survival than cancer patients in the general population [16, 17], and in part because only patients with stage 1 tumour were recruited in both studies. In a consecutive case study of 550 men with testicular cancer seen at a tertiary referral centre, between half and a quarter of testicular cancer patients had metastatic disease and there was clear reduction of the number of patients presenting with more advanced disease overtime [18].

In a setting similar to this study, no difference in survival was found between rich and poor colorectal cancer patients who took part in a large clinical trial [19], when patients were given equal protocol-based treatment. One could argue that the inclusion of patients in clinical studies may lead to selection bias, and that fewer patients are included from deprived areas, perhaps due to severe co-morbidity. However, the distribution of the study patients by deprivation quintiles was very similar to that seen among all cancer patients in the general population. Moreover, information on socioeconomic status was not known to the investigators at the time of entry to the study; it was only derived later from the postcode of the patients at time of diagnosis. It is possible that the deprivation gap observed in the general population is mostly due to differential distribution of tumour stage by deprivation, with higher proportion of the advanced stage 3 disease among the more deprived patients, while only patients with stage 1 were included in these studies. However, there has been continuous improvement in survival since the 1980s, both among elderly and deprived patients while chemotherapy treatment remained largely the same. A likely explanation is that 
more patients are diagnosed at the early stage due to greater awareness of testicular cancer [20]. Several UK centres have also reported fewer patients presenting with advanced disease [21]. Another potential explanation of the 'deprivation gap' seen in the general population could be explained by deprived patients presenting with many comorbid conditions that could affect cancer outcome [22], an example of such more atypical advanced presentations in situations of social deprivation was reported by Mulatero, et al. [23]. However in the context of the relatively young patient population (around $70 \%$ less than 40 years of age) co-morbidity seems like a less probable explanation.

Our findings, based on data collected prospectively in two national clinical studies suggest, that given equal treatment, survival from testicular cancer, at least for stage I disease, which comprise more than half of all cases, with which high percentage of patients are diagnosed, does not depend on socio-economic deprivation. This implies that the deprivation gap seen in the general population is more likely to be due to differences in access to treatment than to patient related factors.

\section{Conflict of interest statement}

None declared 


\section{References}

[1] Coleman MP, Rachet B, Woods LM et al. (2004) Trends and socioeconomic inequalities in cancer survival in England and Wales up to 2001. Br J Cancer 90: $1367-1373$

[2] Kogevinas M, Porta M (1997) Socioeconomic differences in cancer survival: a review of the evidence. In: Social inequalities and cancer. (IARC Scientific Publications No. 138). Kogevinas M, Pearce N, Susser M, Boffetta P (eds) pp 177-206. International Agency for Research on Cancer: Lyon

[3] Coleman MP, Babb P, Mayer D, Quinn MJ, Sloggett A (1999) Cancer survival trends in England and Wales 1971-1995: deprivation and NHS region (CDROM). Office for National Statistics London, Office for National Statistics.

[4] Nur U, Rachet B, Mitry E, Cooper N, Coleman MP (2008) Survival from testicular cancer in England and Wales up to 2001. Br J Cancer 99: S80-S82

[5] Woods LM, Rachet B, Coleman MP (2006) Origins of socio-economic inequalities in cancer survival: a review. Ann Oncol 17: 5-19

[6] Read G, Stenning SP, Cullen MH et al. (1992) Medical-Research-Council Prospective-Study of Surveillance for Stage-I Testicular Teratoma. Journal of Clinical Oncology 10: 1762-1768

[7] Oliver RTD, Mason MD, Mead GM et al. (2005) Radiotherapy versus singledose carboplatin in adjuvant treatment of stage I seminoma: a randomised trial. Lancet 366: 293-300

[8] Carstairs V (1995) Deprivation indices: their interpretation and use in relation to health. J Epidemiol Community Health 49: S3-S8

[9] Woods LM, Rachet B, Coleman MP (2005) Choice of geographic unit influences socioeconomic inequalities in breast cancer survival. $\mathrm{Br} J$ Cancer 92: $1279-1282$

[10] Office of the Deputy Prime Minister (2004) The English Indices of deprivation 2004-summary

(revised). http://www.communities.gov.uk/archived/publications/communities/indicesdepr ivation, accessed 21 Sep 2010

[11] National Assembly for Wales (2000) Welsh index of multiple deprivation. Cardiff, National Assembly for Wales

[12] Estève J, Benhamou E, Croasdale M, Raymond L (1990) Relative survival and the estimation of net survival: elements for further discussion. Stat Med 9: 529538

[13] Cancer Research UK Cancer Survival Group (2004) Life tables for England and Wales by sex, calendar period, region and deprivation. www.lshtm.ac.uk/ncdeu/cancersurvival/tools/strelandlifetables.htm, London School of Hygiene \& Tropical Medicine.

[14] Grizzle JE, Starmer CF, Koch GG (1969) Analysis of categorical data by linear models. Biometrics 25: 489-504

[15] National Institute for Clinical Excellence (2002) Improving Outcomes in Urological Cancers: The manual. http://www.nice.org.uk/nicemedia/live/10889/28771/28771.pdf, accessed 27 Jul 11 A.D.

[16] Sorbye H, Pfeiffer P, Cavalli-Bjorkman N et al. (2009) Clinical Trial Enrollment, Patient Characteristics, and Survival Differences in Prospectively Registered Metastatic Colorectal Cancer Patients. Cancer 115: 4679-4687 
[17] Mayers C, Panzarella T, Tannock IF (2001) Analysis of the prognostic effects of inclusion in a clinical trial and of myelosuppression on survival after adjuvant chemotherapy for breast carcinoma. Cancer 91: 2246-2257

[18] Bhardwa JM, Powles T, Berney D, Baithun S, Nargund VH, Oliver RTD (2005) Assessing the size and stage of testicular germ cell tumours: 1984-2003. Bju International 96: 819-821

[19] Nur U, Rachet B, Coleman MP et al. (2008) No socioeconomic inequalities in colorectal cancer survival within a randomised clinical trial. $\mathrm{Br}$ J Cancer 99: 1923-1928

[20] Huddart RA (2008) Survival from testicular cancer in England and Wales up to 2001 - Clinical commentary. Br J Cancer 99: S83-S85

[21] Bhala N, Coleman JM, Radstone CR et al. (2004) The management and survival of patients with advanced germ-cell tumours: Improving outcome in intermediate and poor prognosis patients. Clinical Oncology 16: 40-47

[22] Gritz ER, Demark-Wahnefried W (2009) Health Behaviors Influence Cancer Survival. Journal of Clinical Oncology 27: 1930-1932

[23] Mulatero C, Brogan G, Oliver RTD (2000) Advanced testicular cancer presenting with phlegmasia cerulea dolens. Postgraduate Medical Journal 76: $234-236$ 


\section{Figures}

\section{Figure 1}

Distribution of patients in the two clinical studies (TE04 and TE19).

\section{Figure 2}

Relative survival (\%) up to ten years by deprivation category, in the study and the general population of England and Wales (1984-2001).

\section{Tables}

\section{Table 1}

Number (\%) of men with testicular cancer by deprivation group

\section{Table 2}

Relative survival (\%) by deprivation category, and deprivation gap (\%) at five and ten years after diagnosis 
Table 1: Number (\%) of men with testicular cancer by deprivation group

(a) Study population (1984-2001)

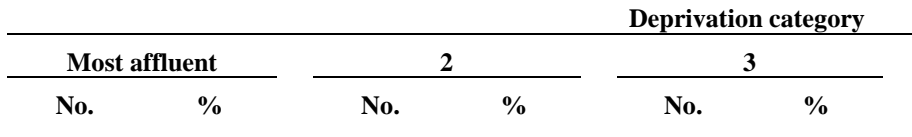

349

21.7

330

No. of patients

Trial

TE04
TE19

$131 \quad 22.7$

TE19

Age at diagnosis

Less than 40

40-49

50 and over

218

21.2

$\begin{array}{ll}124 & 21.5 \\ 206 & 20.0\end{array}$

340

21.2 No.

No. 4

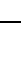
No No.

No of patients No.

1606

100.0

$\mathbf{5 7 8} \quad 36.0$

$1028 \quad 64.0$

$\begin{array}{lll}212 & 20.6 & 225\end{array}$

1.9

$\begin{array}{ll}67 & 16.2\end{array}$

16.2

$1125 \quad 70.0$

$329 \quad 20.5$

$152 \quad 9.5$

(b) General population of England and Wales (1984-2001)

$\begin{array}{lrrrrrrrrrrrr}\text { No. of patients } & \mathbf{5 3 1 3} & \mathbf{2 1 . 9} & \mathbf{4 9 3 2} & \mathbf{2 0 . 3} & \mathbf{4 9 8 3} & \mathbf{2 0 . 5} & \mathbf{4 9 5 8} & \mathbf{2 0 . 4} & \mathbf{4 0 7 8} & \mathbf{1 6 . 8} & \mathbf{2 4} \mathbf{2 6 4} & \mathbf{1 0 0 . 0} \\ \text { Age } & & & & & & & & & & & & \\ \quad \text { Less than 40 } & 3327 & 20.2 & 3268 & 19.8 & 3428 & 20.8 & 3529 & 21.4 & 2939 & 17.8 & \mathbf{1 6} \mathbf{4 9 1} & 68.0 \\ \mathbf{4 0 - 4 9} & 1221 & 26.2 & 1016 & 21.8 & 915 & 19.6 & 856 & 18.4 & 651 & 14.0 & \mathbf{4 6 5 9} & 19.2 \\ \mathbf{5 0} \text { and over } & 765 & 24.6 & 648 & 20.8 & 640 & 20.6 & 573 & 18.4 & 488 & 15.7 & \mathbf{3 1 1 4} & 12.8\end{array}$


Table 2: Relative survival (\%) by deprivation category, and deprivation gap (\%) at five and ten years after diagnosis

\begin{tabular}{|c|c|c|c|c|c|c|c|c|c|c|c|c|c|c|c|}
\hline \multirow[b]{4}{*}{ Deprivation } & \multirow[b]{4}{*}{ Patients } & \multirow[b]{4}{*}{ Deaths } & \multicolumn{6}{|c|}{ Five-year survival } & & \multicolumn{6}{|c|}{ Ten-year survival } \\
\hline & & & \multicolumn{3}{|c|}{ Crude survival } & \multicolumn{3}{|c|}{ Relative survival } & \multirow[b]{3}{*}{ Deaths } & \multicolumn{3}{|c|}{ Crude survival } & \multicolumn{3}{|c|}{ Relative survival } \\
\hline & & & & \multicolumn{2}{|c|}{$95 \% \mathrm{CI}$} & & \multicolumn{2}{|c|}{$95 \%$ CI } & & \multicolumn{3}{|c|}{$95 \% \mathrm{CI}$} & & \multicolumn{2}{|c|}{$95 \%$ CI } \\
\hline & & & & Lower & Upper & & Lower & Upper & & & Lower & Upper & & Lower & Upper \\
\hline \multicolumn{16}{|c|}{ populations (1984-2001) } \\
\hline luent & 349 & 8 & 97.7 & 95.5 & 98.8 & 98.8 & 95.8 & 99.7 & 11 & 96.8 & 94.4 & 98.2 & 98.2 & 95.2 & 99.3 \\
\hline 2 & 330 & 12 & 96.4 & 93.7 & 97.9 & 97.3 & 94.5 & 98.7 & 17 & 94.6 & 91.4 & 96.6 & 96.4 & 92.9 & 98.2 \\
\hline 3 & 340 & 1 & 99.7 & 97.9 & 100.0 & 100.0 & - & - & 7 & 97.7 & 95.1 & 98.9 & 99.2 & 93.3 & 99.9 \\
\hline 4 & 337 & 1 & 99.7 & 97.9 & 100.0 & 100.0 & 100.0 & - & 5 & 98.3 & 95.8 & 99.3 & 99.5 & 90.4 & 100.0 \\
\hline rived & 250 & 4 & 98.4 & 95.8 & 99.4 & 99.8 & - & 100.0 & 7 & 97.0 & 93.7 & 98.6 & 99.8 & - & 100.0 \\
\hline ion gap & & & & & & 1.4 & & & & & & & 2.2 & & \\
\hline \multicolumn{16}{|c|}{ ral population of England and Wales (1984-2001) } \\
\hline luent & 5313 & 350 & 93.4 & 92.7 & 94.0 & 95.3 & 94.6 & 95.9 & 459 & 91.1 & 90.3 & 91.9 & 94.8 & 94.0 & 95.4 \\
\hline 2 & 4932 & 351 & 92.9 & 92.1 & 93.5 & 94.9 & 94.2 & 95.6 & 448 & 90.7 & 89.8 & 91.5 & 94.5 & 93.7 & 95.2 \\
\hline 3 & 4983 & 423 & 91.5 & 90.7 & 92.2 & 94.2 & 93.4 & 94.9 & 540 & 88.9 & 87.9 & 89.7 & 93.3 & 92.4 & 94.1 \\
\hline 4 & 4958 & 441 & 91.1 & 90.2 & 91.8 & 93.8 & 93.0 & 94.5 & 567 & 88.1 & 87.2 & 89.0 & 93.3 & 92.4 & 94.1 \\
\hline rived & 4078 & 469 & 88.4 & 87.4 & 89.4 & 91.6 & 90.6 & 92.5 & 577 & 85.4 & 84.3 & 86.5 & 91.1 & 90.0 & 92.1 \\
\hline tion gap & & & & & & $-3.1 *$ & & & & & & & $-3.1^{*}$ & & \\
\hline
\end{tabular}

*Statistically significant at $1 \%$.

- unavailable estimates due to small number of deaths in the specific time interval 